\title{
Successive acquisitions and extinctions of discrete bar-pressing in monkeys and rats ${ }^{1}$
}

JOHN W. DAVENPORT, Regional Primate Research Center, University of Wisconsin, Madison, Wis. 53706

Five stumptail monkeys and eight rats were given 17 acquisition-extinction cycles in a discrete-trial fixed-ratio (FR 5) barpressing situation. Progressive increases in rates of acquisition and extinction were found in both species, and the monkeys displayed higher relative mates of extinction than the rats over all cycles. After the first three extinctions, however, four of the monkeys showed progressively elevated extinction asymptotes rather than the increasing incidence of nonresponse trials which would normally be expected. Terminal performances consisted of one-trial reacquisition in both species, relative extinction (100\% decline to asymptote) in one or two trials in the monkeys, and extinction in three to six trials in the rats.

Although simple learning paradigms such as the acquisition of instrumental reward learning have generally shown little promise as tasks for revealing species differences in learning (Warren, 1965), the extensive Russian research reported by Voronin (1962), various findings at the Bryn Mawr laboratory (e.g., Bitterman, 1965; Gonzalez, Holmes, \& Bitterman, 1967), and many informal observations at our Wisconsin laboratory have indicated that the study of inhibitory processes within such simple paradigms may be fruitful in disclosing behavioral differences which are clearly correlated with phyletic position. Among various inhibitory phenomena (extinction, differential conditioning, conditioned inhibition, etc.), species comparison in successive acquisitions and extinctions is particularly interesting in view of Voronin's (1962, p. 166) finding of a negative correlation between phyletic position and the number of acquisition-extinction cycles to one-trial extinction, and because of the relevance of this experimental treatment to successive discriminations and reversals in which learning sets are studied.

In the present experiment, albino rats and stumptail monkeys (Macaca speciosa) were compared over 17 acquisition-extinction cycles in a discrete-trial fixed-ratio (FR 5) retractable lever situation. Conditions were arranged so as to provide a detailed view of successive spontaneous recoveries, the effects of the first reinforced trial in each of the acquisition periods, and the effects of the first nonreinforced trial in each of the extinctions.

\section{SUBJECTS}

The Ss were eight adult male albino rats of the Holtzman strain and six (three male, three female) adolescent stumptail monkeys, one of which had to be dropped during the experiment because of illness. All Ss were experimentally naive. They were maintained at approximately $85 \%$ of free-feeding weights throughout the experiment.

APPARATUS
The apparatuses were Lehigh Valley 1317 (rat) and 1316 (monkey) operant test chambers, each equipped with two retract. able levers, only the right-hand one of which was used. Each chamber was also equipped with a center key mounted between the lever positions, a houselight, and a feeder for dispensing pellets (45-mg Noyes pellets for the rats, $97 \cdot \mathrm{mg}$ sucrose pellets for the monkeys). All functions of the chambers were automatically controlled by programming and recording equipment in an adjacent room.

\section{PROCEDURE}

In pretraining (Days 1-3), each $S$ was given one session of magazine training (50 pellets on a variable-interval 30 -sec schedule), a second session of lever shaping (50 continuously reinforced responses in a free-operant mode), and a third session of discrete-trial key training ( 50 trials in which presentation of the lever and houselight was contingent upon S's pressing the key, and a single lever response was reinforced by one pellet).

Acquisition (A-1) training was given in 30-trial sessions on Days 4-8 and the first 10 trials of Day 9. In these and all subsequent reacquisition sessions, completion of five lever responses (after trial-initiation by a keypress) was required in each trial for reinforcement (one pellet). Trials were separated by variable-length intertrial intervals of total darkness averaging $30 \mathrm{sec}$. At the end of each intertrial interval the key was illuminated, and S's key response extinguished the key light and presented the lever and houselight as on Day 3. The fifth lever response produced retraction of the lever and activated the pellet dispenser, after which a 5-sec magazine period (continuation of the houselight) terminated the trial. No time limit was imposed on trial initiation or lever responding on rewarded trials.
The first extinction (E-1) began on the 11 th trial of Day 9 with no change in the intertrial interval, and continued through Trial 30 of Day 9 and three additional 30-trial sessions (Days 10-12). In these and all subsequent extinction sessions, trial conditions were identical to those of reinforced trials except that the pellet dispenser was not activated and time limits were imposed. If no key response was made within $15 \mathrm{sec}$, the key light was ex tinguished and the lever and houselight were presented automatically; trials were terminated if completion of the fixed ratio did not occur within $60 \mathrm{sec}$ after lever presentation.

Following the $160 \mathrm{~A}-1$ and $110 \mathrm{E}-1$ trials on Days 4-12, 16 additional acquisitionextinction cycles were administered on Days 13-34, with extinctions always starting after a normal intertrial interval in the midst of a session. In the first six cycles after E-1, 30-trial reacquisition sessions (A-2 through A-7) alternated with extinction sessions(E-2 through E-7), which began with the final 10 trials of a given reacquisition phase and were run for at least 20 nonreinforced trials and until individual $S s$ met a criterion of three consecutive trials in which the FR 5 was not completed, or a limit of 100 extinction trials, whichever occurred first. When it became clear that reacquisition required only a few trials in all Ss and that some of the monkeys would seldom meet the three-trial extinction criterion within 100 trials, the testing schedule was altered. Thus, in the final 10 cycles (Days 25-34), single sessions consisting of only 10 reacquisition trials and 20 extinction trials were administered.

Measures taken on each trial throughout the experiment were the key latency (equivalent to the duration of key illumination), latency of the first lever response (measured from the onset of the key response), and ratio-completion time (from the first to the fifth lever response). These measures were converted to reciprocals and referred to as key speed, start speed, and ratio speed, respectively.

\section{RESULTS AND DISCUSSION}

Both species required well over 100 trials to reach speed asymptotes in acquisition. Speeds were higher for the rats than the monkeys over most of the acquisition period (for start speed, $\mathrm{F}=7.42 ; \mathrm{df}=1 / 11$; $\mathrm{p}<.05)$ and in subsequent reacquisitions.

Start speeds for the last 10 acquisition trials, the first extinction period (E-1), and all remaining acquisition-extinction cycles are shown in Fig. 1. In this figure, trials are in blocks of three, except for the 1st, 2nd, and 12 th of each 30-trial series, which are plotted separately in order to reveal spontaneous recovery and changes following single rewarded or nonrewarded trials. After E-1 and A-2, which are shown in their entirety, data from only 19 nonrewarded 


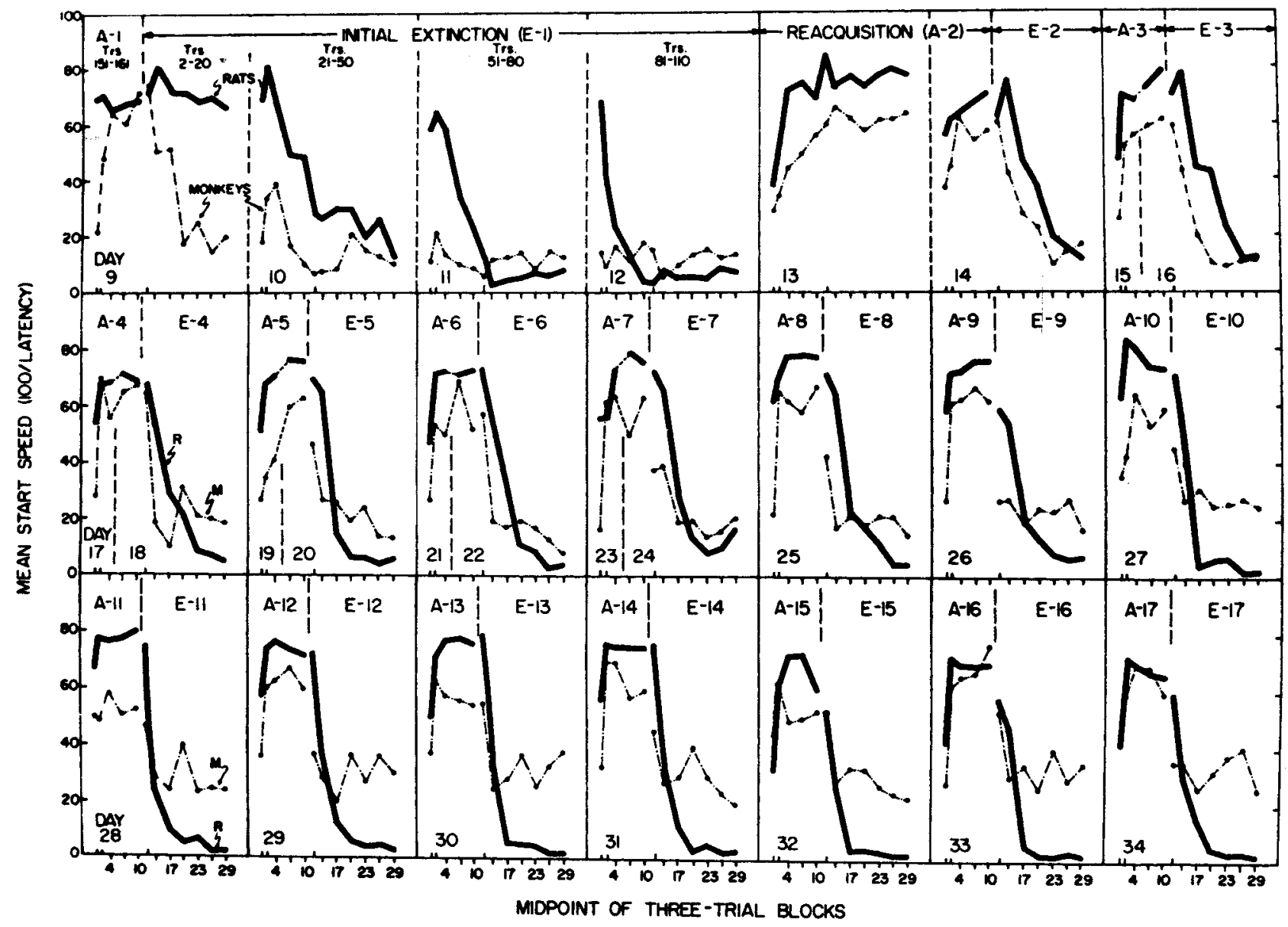

Fig. 1. Mean start speeds for the two species at the end of acquisition and overall subsequent acquisition-extinction cycles.

trials following the first trial of each extinction phase are shown, even though the Ss were given additional extinction trials in E-2 through E-7. In the 40-trial reacquisitions, A-3 through A-7, only the first five and last five trials are plotted, with the intervening 30 trials omitted. Thereafter (Cycles 8 through 17), data from 10 rewarded and all 20 nonrewarded trials in each cycle are plotted. In all acquisitions, the final three-trial block is composed of the last two acquisition trials and combined with the first extinction trial.

Performance in E-1 was so different for the two species that statistical analysis was unnecessary. Within the first 10 extinction trials, all five of the monkeys dropped to an apparent extinction asymptote of start speed, while all eight of the rats remained near their acquisition asymptotes over the first (20-trial) E-1 session. In subsequent E-1 sessions, high amounts of spontaneous recovery and resistance to extinction were shown by the rats, whereas the monkeys showed no spontaneous recovery on the first trial of Sessions 10,11, and 12, and only slight indications of spontaneous recovery on subsequent trials.
Both species showed progressive increases in rate of reacquisition, and by A-11 a single rewarded trial was sufficient to bring start speeds of all Ss to reacquisition asymptotes. Spontaneous recovery continued to be much higher in the rats than the monkeys in terms of speed increases from the end of an extinction period to the first trial of a subsequent reacquisition. In this connection it should be noted that spontaneous regression, defined as the speed decrement from the end of a rewarded session to the first trial of a subsequent rewarded session, was substantially greater for the monkeys than the rats in acquisition (see Day 9 , Fig. 1) and in all of the two-session reacquisitions except A-2.

The successive extinctions produced a surprising effect in the monkeys - a progressive elevation of apparent extinction asymptotes to an average start-speed level that was about $50 \%$ of reacquisition asymptotes over the last few extinctions. This effect was shown by four of the monkeys in all three speed measures and resembled a "nonextinction effect" which stumptail monkeys have repeatedly displayed in unpublished research in our laboratory involving homogeneous chaining behavior in an eight-pushbutton apparatus. In contrast, one of the monkeys (included in Fig. 1) and all of the rats showed "complete" extinction in the form of nonresponse trials in the later extinctions.

In terms of relative rate of extinction, however, the monkeys were clearly superior to the rats. That is, defining extinction rate as the percentage of the total speed decrement from the end of a reacquisition period to an extinction asymptote resulting from the first nonrewarded trial, the monkeys consistently showed a higher extinction rate than the rats over the successive extinctions, as they had shown in E-1. On Extinctions E-9, E-12, and E-17, all five monkeys displayed one-trial extinction (100\% decrement on Trial 12), and two-trial extinction on most cycles beyond E-3, whereas the rats usually showed only small decrements or slight increments in speed from Trial 11 to Trial 12 over all cycles. In this sense the results from the successive extinctions are consistent with Voronin's (1962) findings and the generalization that sensitivity to nonreward increases with phyletic status. 


\section{REFERENCES}

BITTERMAN, M. E. Phyletic differences in learning. American Psychologist, 1965, 20 , 396-410.

GONZALEZ, R. C., HOLMES, N. K., \& BITTERMAN, M. E. Asymptotic resistance to extinction in fish and rat as a function of interpolated retraining. Joumal of Comparative \& Physiological Psychology, 1967, 63, 342-344.

VORONIN, L. G. Some results of comparativephysiological investigations of higher nervous activity. Psychological Bulletin, 1962, 59, 161-195.
WARREN, J. M. Primate learning in comparative perspective. In A. M. Schrier, H. F. Harlow, and F. Stollnitz (Eds.), Behavior of nonhuman primates. Vol. 1. New York: Academic Press, 1965. Pp. 249-281.

\section{NOTE}

1. Supported by Grant FR-00167 from the National Institutes of Health. I am grateful to Robert Woodworth, William Hagquist, and Diane Eich for their assistance.

\section{The placement of shocking electrodes for escape and avoidance learning in fish}

\author{
FRANK T. SEVERIN, Saint Louis \\ University, St. Louis, Mo. 63103
}

Measurements were made to determine whether the voltage gradient in the body of a fish swimming between two vertical electrically charged plates would vary with the animal's orientation. The voltage gradient in anesthesized goldfish positioned perpendicular to the electrodes was found to be five times greater than when oriented parallel to them. A suggestion is made for minimizing this differential in avoidance-learning apparatus.

An experimental situation for small aquatic animals described by Horner, Longo, \& Bitterman (1961) and Bitterman (1966) has stimulated interest in the use of fish in psychological laboratories for the study of escape and avoidance learning. One feature of this apparatus which has been widely imitated is to place the shocking plates in the walls of the training tank.

Judging from the voltage gradient between electrodes set either at the sides or

Table 1

Gradient in Volts Measured in the Bodies of Goldfish Oriented Perpendicular with and Horizontal to Two Vertical Electrodes

\begin{tabular}{lcr} 
& \multicolumn{2}{c}{ Orientation of Fish } \\
\cline { 2 - 3 } & Perpendicular & Horizontal \\
\hline Fish A & 1.6 & 0.3 \\
Fish B & 1.6 & 0.3 \\
Fish C & 1.6 & 0.3 \\
Electrodes without Fish 1.7 & 0.3 \\
\hline
\end{tabular}

the ends of a tank, the shock delivered to a fish should vary in intensity depending upon its orientation at the moment. When positioned perpendicular to the electrodes, the voltage drop along the length of its body should be greater than across its narrow dimension when swimming parallel to them. The present investigation was undertaken to verify this differential.

\section{METHOD}

Three goldfish of $5.5 \mathrm{~cm}$ standard length served as Ss. The apparatus consisted of two vertical stainless steel plates, $15 \mathrm{~cm}$ square, placed parallel to each other, $15 \mathrm{~cm}$ apart, in a plastic container of water. A potential of $8 \mathrm{~V}$ was applied across these plates by means of a battery in series with a variable resistor. Two sets of needle electrodes, $10 \mathrm{~cm}$ in length, were fashioned from 20-gauge silver wire and fixed rigidly in $3 \times 3 \times 14 \mathrm{~cm}$ holders. The $5 \cdot \mathrm{cm}$ distance between one pair of electrodes corresponded to the length of a goldfish. The $1.1 \cdot \mathrm{cm}$ spacing of the other pair represented the narrow dimension of the fish. The ends of the electrodes to be immersed in water were sharpened to a point and bent into small hooks parallel to each other. To inhibit polarization, the silver wires were chlorided by connecting them through a battery to a sheet of silver in a $5 \%$ sodium chloride solution (Hill, 1950). Voltage measurements were made with a Sylvania vacuum tube polymeter, Model 303.

Each set of needle electrodes was immersed 4 to $5 \mathrm{~cm}$ in the water, midway between the metal plates, with its holder perpendicular to them. The voltage drop across the electrodes was then determined. Throughout the experiment, 10 readings were taken for each measurement and the average value accepted. The electrodes were tested for polarization before and after each set of 10 readings by placing them in a sodium chloride solution while attached to a battery to see whether they would support a steady current of a fraction of a milliampere for $60 \mathrm{sec}$ (Hill, 1950). A goldfish, anesthesized with MS 222 (Tricainmethanesulfonate), was attached by the snout and region of the caudal peduncle to the first pair of needle electrodes and placed perpendicular to the plates midway between them. Readings were taken of the voltage gradient along the length of the fish. The second pair of electrodes was then hooked skin deep across the narrow dimension of the fish and similar readings taken, with the fish positioned parallel to the plates. These measurements were repeated with two additional goldfish. RESULTS AND CONCLUSION

Since the shocking plates used in this experiment replicate the condition of electrodes placed either in the walls or the ends of an avoidance training tank, it can be seen that the voltage gradient in the body of a fish will depend upon its own alignment. Positioned perpendicularly to the shocking plate, the voltage drop measured along the length of a fish was five times greater than across its narrow dimension when oriented parallel to them. These findings confirm a subjective experience which served as the starting point for this investigation. While experimenting with electrodes set in the sides of a smaller tank, the author placed his hand in the water between them. When held with the axis between the thumb and middle finger perpendicular to the electrically charged plates, a stinging sensation was felt in abrasions on the joints of these two fingers. The pain ceased whenever the hand was rotated $90 \mathrm{deg}$. A fish generally maintains a level position in the water and, in diving or surfacing, describes a small angle compared with the $360 \mathrm{deg}$ it is free to turn in the horizontal plane. Electrodes placed at the bottom of the tank and near the surface of the water should minimize differences in voltage gradient due to the animal's orientation.

\section{REFERENCES}

BITTERMAN, M. E. Animal learning. In J. Sidowski (Ed.), Experimental methods and instrumentation in psychology. New York: McGraw-Hill, 1966.

HILL, D., \& PARR, G. (Eds.) Electroencephalography. London: MacDonald, 1950.

HORNER, J. L., LONGO, N., \& BITTERMAN, M. E. A shuttie box for the fish and a control circuit of general applicability. American Journal of Psychology, 1961, 74, 114-120. 